\title{
Primär kutane B-Zell-Lymphome
}

\section{Primary Cutaneous B-Cell Lymphomas}

O. Inhoff, E. Dippel

Hautklinik/Hautttumorzentrum Rheinpfalz, Klinikum der Stadt Ludwigshafen

\section{Lernziele}

$\nabla$

Kenntnisse über primär kutane B-Zell-

Lymphome:

- Terminologie und Klassifikation

- Klinik, Histologie und Diagnosestellung

- Stadieneinteilung

- Therapie und Prognose

\section{Einleitung}

Primär kutane Lymphome (cutaneous lymphomas: CL) entstehen definitionsgemäß in der Haut und zeigen zum Zeitpunkt der Diagnosestellung nach abgeschlossener Durchuntersuchung keine extrakutane Beteiligung. Nach den gastrointestinalen MALT-Lymphomen (MALT: mucosa associated lymphatic tissue) bilden die primär kutanen Lymphome die zweithäufigste Gruppe der extranodalen Non-Hodgkin-Lymphome (NHL) mit einer geschätzten Inzidenz von einer Neuerkrankung pro 100000 Einwohner pro Jahr. CL stellen eine heterogene Gruppe neoplastischer lymphoproliferativer Erkrankungen dar, mit Unterschieden im klinischen und histologischen Bild, in der Pathogenese und Prognose. 73\% der CL können den kutanen T-Zell-Lymphomen (cutaneous T-cell lymphomas: CTCL), 22\% den kutanen B-Zell-Lymphomen (cutaneous B-cell lymphomas: $\mathrm{CBCL}$ ) und < $10 \%$ hämatologischen Vorläuferneoplasien zugeordnet werden [1,2]. Im Folgenden sollen die Klassifikation, Stadieneinteilung, Prognose und Therapie der CBCL näher betrachtet werden.

\section{Terminologie und Klassifikation primär kutaner B-Zell-Lymphome \\ $\nabla$}

In gesunder Haut finden sich nur sehr wenige BLymphozyten, und erst in den letzten 2 Jahrzehnten wurde klar, dass einige seltene Subtypen von B-Non-Hodgkin-Lymphomen sich ausschließlich in der Haut präsentieren können. CBCL sind deut- lich seltener als CTCL und repräsentieren annähernd 20-25\% der primär kutanen Lymphome. Hinsichtlich Terminologie und Klassifikation der CBCL bestand jahrelang Uneinigkeit bis 2005 durch Zusammenarbeit von WHO (World Health Organization) und EORTC (European Organisation for Research and Treatment of Cancer) eine neue, die sogenannte WHO-EORTC-Klassifikation entstand. Bis dato führten die Organisationen 2 unterschiedliche Klassifikationssysteme. Dabei wurden Terminologie und Klassifikation der $\mathrm{CBCL}$ vereinheitlicht und neben der Morphologie auch das klinische Verhalten berücksichtigt. Die jüngste Version der WHO-Klassifikation von 2008 verändert die Bewertung der CBCL nicht.

Die WHO-EORTC-Klassifikation berücksichtigt 3 Haupttypen von CBCL:

\section{Indolentes klinisches Verhalten}

- primär kutane Marginalzonen B-Zell-Lymphome (primary cutaneous marginal zone B-cell lymphoma: PCMZL)

- primär kutane Keimzentrumslymphome (primary cutaneous follicle center lymphoma: PCFCL)

- follikulär

- follikulär und diffus

- diffus

\section{Intermediäres klinisches Verhalten}

- primär kutane diffus-großzellige B-Zell-Lymphome (leg type) (primary cutaneous diffuse large B-cell lymphoma, leg type: PCLBCL, LT)

- primär kutane diffus-großzellige B-Zell-Lymphome (other) (primary cutaneous diffuse large B-cell lymphoma, other: PCLBCL, other) - primär kutane intravaskuläre großzellige B-Zell-Lymphome (intravascular large B-cell lymphoma: IVLBCL)

Das PCMZL und das PCFCL zählen mit einer 5-Jahres-Überlebensrate von $\geq 95 \% \mathrm{zu}$ den indolenten Non-Hodgkin-Lymphomen. Das PCLBCL, LT unterscheidet sich hiervon grundsätzlich mit einer 5Jahres-Überlebensrate von nur 55\% [1]. Die indolenten $\mathrm{CBCL}$ (PCMZL/PCFCL) werden aufgrund ihrer morphologischen Ähnlichkeiten auch als kutane Äquivalente der MALT-Lymphome betrachtet
VNR

2760512011060003972

Bibliografie

DOI http://dx.doi.org/ 10.1055/s-0030-1256016 Akt Dermatol 2011; 37: 47-56 (c) Georg Thieme Verlag KG Stuttgart · New York ISSN 0340-2541

Korrespondenzadresse Dr. med. Oliver Inhoff Funktionsoberarzt Hautklinik/Hauttumorzentrum Rheinpfalz

Klinikum Ludwigshafen Bremserstr. 79 67063 Ludwigshafen inhoffo@klilu.de 
und von einigen Autoren in Anlehnung an diesen Begriff als SALT-Lymphome (SALT: skin associated lymphoid tissue) bezeichnet [3].

Neben dem PCLBCL, LT können sich sehr selten auch andere Typen primär kutaner diffus-großzelliger B-Zell-Lymphome primär an der Haut manifestieren. Hier muss zunächst immer eine Beteiligung der Haut im Rahmen eines extrakutanen Lymphoms ausgeschlossen werden. Das primär kutane intravaskuläre großzellige B-ZellLymphom stellt eine weitere sehr seltene Entität dar.

Das primär kutane Marginalzonen B-Zell-Lymphom und das primär kutane Keimzentrumslymphom zählen mit einer 5-Jahres-Überlebensrate von $\geq 95 \%$ zu den indolenten Non-Hodgkin-Lymphomen. Das primär kutane diffusgroßzellige B-Zell-Lymphom (leg type) unterscheidet sich hiervon grundsätzlich mit einer 5-Jahres-Überlebensrate von nur $55 \%$.

\section{Diagnose}

Mit dem Ziel, die Ausbreitungsdiagnostik und die Therapie der CBCL zu vereinheitlichen, wurden 2008 die EORTC-ISCL-Konsensusempfehlungen (ISCL: International Society for cutaneous Lymphomas) für das Management von CBCL veröffentlicht [4]. Eine atraumatisch entnommene Probebiopsie adäquater Größe ist unabdingbar, wenn klinisch ein CBCL vermutet wird. Neben der Beurteilung der Morphologie der neoplastischen B-Zell-Population und dem Wachstumsmuster des malignen Infiltrats ist eine Immunhistochemie für die korrekte Diagnosestellung notwendig. Der molekulare Nachweis einer klonalen Immunglobulin-Schwerkettenumlagerung kann zusätzlich hilfreich sein, muss aber stets in Verbindung mit dem klinischen Bild, der Histologie und der Immunhistologie betrachtet werden. Trotz der zur Verfügung stehenden diagnostischen Möglichkeiten kann im Einzelfall eine definitive Einordnung mitunter Schwierigkeiten bereiten, die Diagnosestellung bleibt dann ggf. dem weiteren Verlauf vorbehalten. Typische differenzialdiagnostische Beispiele hierfür sind die Unterscheidung zwischen den indolenten CBCL und einem reaktiven B-Zell-Pseudolymphom, die Unterscheidung zwischen einem follikulären B-ZellLymphom mit klassisch follikulärem Wachstumsmuster und einem Marginalzonen-Lymphom mit reaktiven Keimzentren oder die Unterscheidung eines follikulären B-Zell-Lymphoms mit diffusem Wachstumsmuster von einem diffus großzelligen B-Zell-Lymphom (leg type).

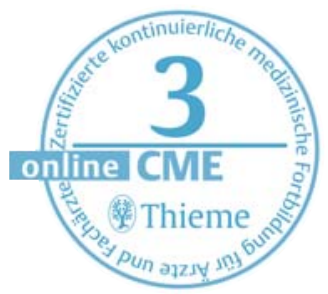

2008 wurden die EORTC-ISCL-Konsensusempfehlungen für das Management von kutanen B-Zell-Lymphomen veröffentlicht.

\section{Klinik und Histologie der CBCL}

$\nabla$

Im Unterschied zu den kutanen T-Zell-Lymphomen zeigen CBCL in der Regel keine epidermale Beteiligung und präsentieren sich als Papeln, Plaques oder Knoten mit derber Konsistenz im Haut- und Unterhautgewebe. Bei kutanen B-ZellLymphomen lassen sich 2 Hauptwachstumsmuster in der histologischen Übersichtsvergrößerung identifizieren. Als Prototyp bei den indolenten CBCL findet sich ein sogenanntes B-Zell-Muster, ein noduläres Infiltrat dicht stehender Lymphozyten mit einer subepidermalen freien Grenzzone. Das diffuse Wachstumsmuster ist durch eine diffuse Infiltration der gesamten Dermis charakterisiert. Davon abzugrenzen sind pseudolymphomatöse Wachstumsmuster, die eine Mischung aus einem diffusen bandähnlichen T-Zell-Muster und einem nodulären B-Zell-Muster mit einer eher konkaven Grenze und mit interstitieller Ausbreitung zeigen [5].

Als Prototyp bei den indolenten $\mathrm{CBCL}$ findet sich histologisch ein sogenanntes B-Zell-Muster, ein noduläres Infiltrat dicht stehender Lymphozyten mit einer subepidermalen freien Grenzzone.

\section{Primär kutane Marginalzonen \\ B-Zell-Lymphome}

Klinik

Das PCMZL tritt gewöhnlich in der 6. Lebensdekade auf, obgleich auch Fälle bei 17-Jährigen beschrieben sind, mit leichtem Überwiegen bei Männern [6]. Das klinische Bild ist gekennzeichnet durch asymptomatische rote bis violette Papeln (Durchmesser 1-3 cm), Plaques oder Knoten, bevorzugt lokalisiert am Stamm oder den Armen $[1,3]$. Im Gegensatz zum PCFCL findet sich häufiger ein multifokaler Hautbefall. Eine Ulzeration der Läsionen ist unüblich. Es besteht die Tendenz zum kutanen Rezidiv, die extrakutane Beteiligung ist jedoch sehr selten [1]. In einigen Fällen kann eine spontane Abheilung, teils unter Ausbildung einer Anetodermie, beobachtet werden [7].

\section{Histologie}

Beim PCMZL finden sich knotige bis diffuse Infiltrate unter Aussparung der Epidermis. Das Infiltrat zeigt häufig reaktive Keimzentren mit deutlich verbreiteter Marginalzone. Überwiegend zeigen sich kleine Lymphozyten, MarginalzonenB-Zellen (zentrozytenähnliche Zellen), lymphoplasmazytoide Zellen, Plasmazellen und viele reaktive T-Zellen [1]. Die Marginalzonen-B-Zellen exprimieren CD20, CD79a und bcl-2, sind jedoch negativ für CD5, CD10 und bcl-6. Reaktive Keimzentren sind typischerweise bcl-6+, CD10+ und bcl-2-. Es findet sich eine klonale Genumlagerung der Immunglobulin-Schwerketten [1] ( $\bullet$ Abb. 1). 


\section{Primär kutane Keimzentrumslymphome} Klinik

Das Keimzentrumslymphom tritt meist um das 60. Lebensjahr auf, wobei auch hier Fälle bei 20jährigen Patienten beschrieben wurden, mit leichtem Überwiegen beim weiblichen Geschlecht [6]. Es zeigen sich bläulichrote bis rötlichbraune solitäre oder gruppierte Plaques (Durchmesser $>3 \mathrm{~cm}$ ) und Tumoren, bevorzugt lokalisiert am behaarten Kopf, an Stirn, Nacken oder Stamm, selten können diese ulzerieren [1,3]. Multifokaler Hautbefall findet sich nur bei einer kleinen Minderheit der Patienten, ist jedoch nicht mit einer ungünstigeren Prognose assoziiert. Bei ausbleibender Behandlung kommt es zur Größenzunahme der Läsionen über Jahre hinweg, extrakutane Manifestationen sind jedoch selten [1].

\section{Histologie}

Es kann sich ein follikuläres, follikulär und diffuses oder ein diffuses Wachstumsmuster zeigen. Die Epidermis ist beinahe immer ausgespart. Das Infiltrat setzt sich zusammen aus Follikelzentrumszellen mit einer variablen Anzahl von Zentrozyten und Zentroblasten. Es werden PanB-Zell-Antigene exprimiert (CD19, CD20, CD79a), CD5 ist jedoch negativ. Meistens findet sich bcl-6 positiv, bcl-2 ist häufig negativ, die CD10-Expression ist variabel. Es findet sich eine klonale Genumlagerung der Immunglobulin-Schwerketten $[1,8]$. Bei Vorliegen einer Positivität für bcl-2 sollte differenzialdiagnostisch ein nodales Keimzentrumslymphom in Betracht gezogen werden (৫ Abb. 2).

\section{Primär kutanes diffus-großzelliges B-Zell-Lymphom (leg type)}

Klinik

Betroffen sind Menschen höheren Alters, meist im 8. Lebensjahrzehnt, mit leichtem Überwiegen des weiblichen Geschlechts [6]. Am häufigsten findet man rötliche bis bläuliche schnell wachsende Knoten und Tumoren an einem oder an beiden Beinen (Unterschenkel). In 10-15\% finden sich auch Läsionen außerhalb der Beine, was aufgrund der Namensgebung zu einer gewissen Konfusion führen kann [9]. Im Vergleich zu den indolenten Formen primär kutaner B-Zell-Lymphome kommt es beim diffus-großzelligen Typ häufig zu einer extrakutanen Beteiligung.

\section{Histologie}

Charakteristischerweise findet sich ein dichtes diffuses Infiltrat aus großen zentroblastenähnlichen (große Follikelzentrumszellen mit prominenten Nukleoli) Zellen unter Aussparung der Epidermis. Mitosen sind vermehrt sichtbar. Die neoplastischen Zellen exprimieren B-Zell-Marker (z.B. CD19, CD20, CD22 und CD79a). Bcl-2- und Mum-1-Expression sind gewöhnlich positiv, bcl6 ist häufig positiv, die CD10-Färbung ist gewöhnlich negativ $[10,11]$. Ein weiteres diagnostisches

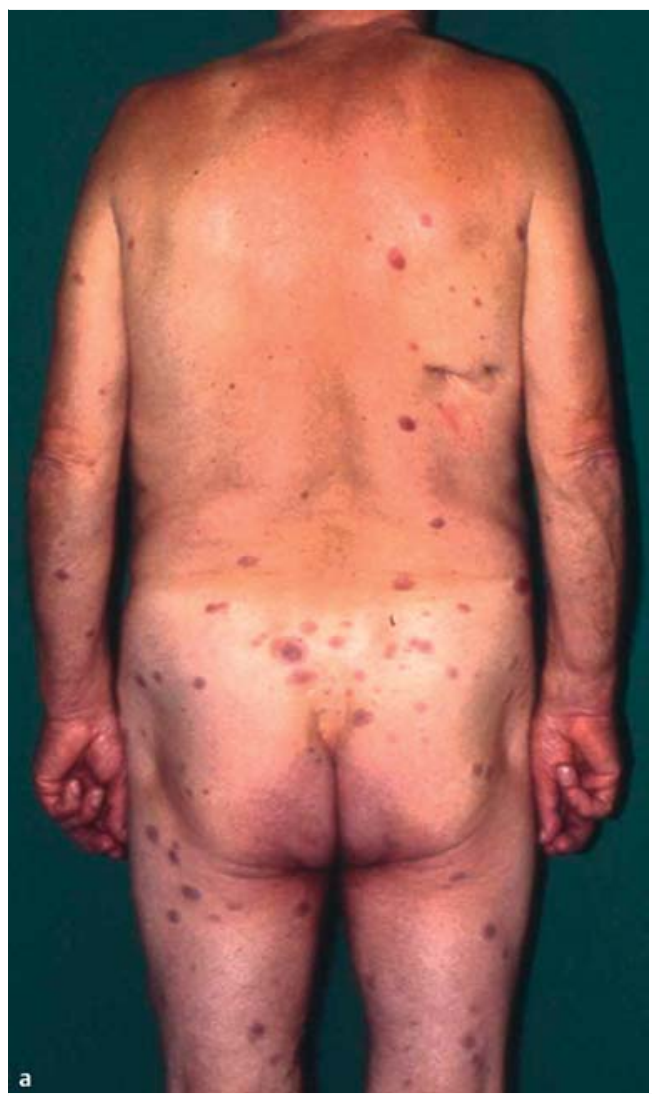

Abb. 1 Primär kutanes Marginalzonenlymphom: Multiple Papeln und Plaques am Körperstamm und den Extremitäten (a). Histologisch zeigen sich knotige bis diffuse Infiltrate überwiegend aus kleinen Lymphozyten und Marginalzonen-B-Zellen unter Aussparung der Epidermis (b). In der Immunhistologie zeigen sich die Tumorzellen bcl-2-positiv (c).
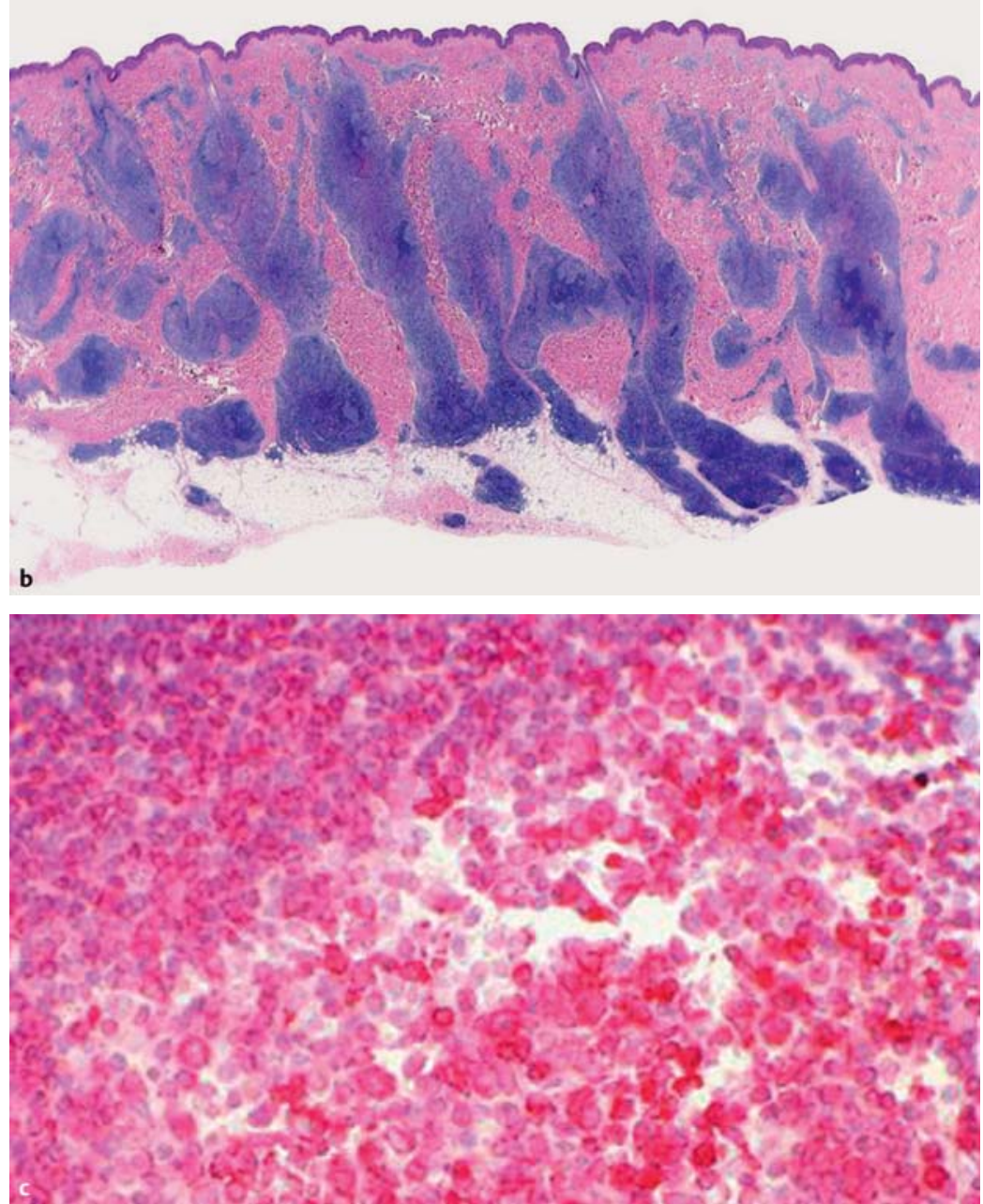
Abb. 2 Primär kutanes Keimzentrumslymphom: Multiple rötliche Plaques lokalisiert an der Kopfhaut (a), histologisch zeigen sich hier follikuläre Infiltrate $(\mathbf{b})$.
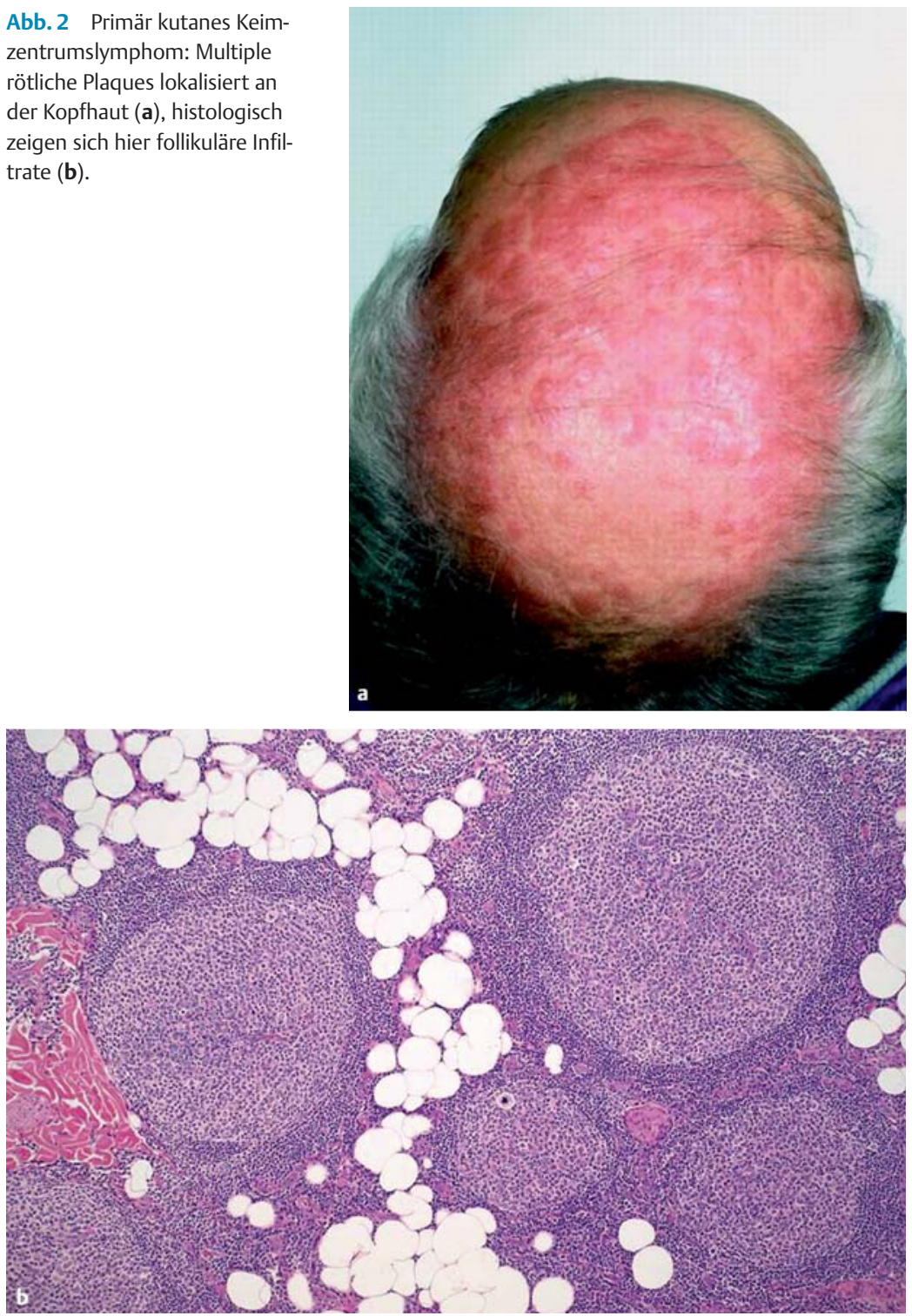

Zeichen ist die klonale Genumlagerung der Immunglobulin-Schwerketten ( $\bullet$ Abb. 3).

Im Vergleich zu den indolenten Formen primär kutaner B-Zell-Lymphome kommt es beim diffus-großzelligen Typ häufig zu einer extrakutanen Beteiligung.

\section{Primär kutanes diffus-großzelliges B-Zell-Lymphom (other)}

Der Begriff PCLBCL (other) bezieht sich auf die seltenen Fälle kutaner großzelliger B-Zell-Lymphome, welche nicht in die Gruppe der PCLBCL (leg type) oder der PCFCL eingeordnet werden können. Es handelt sich um morphologische Varianten des diffus-großzelligen B-Zell-Lymphoms, wie etwa anaplastische oder plasmablastische Subtypen oder T-Zell-/histiozytenreiche großzellige B-Zell-Lymphome. Hier muss zunächst immer eine Beteiligung der Haut im Rahmen eines systemischen Lymphoms ausgeschlossen werden.
Intravaskuläres großzelliges

\section{B-Zell-Lymphom}

Das IVLBCL ist eine seltene Form diffus-großzelliger B-Zell-Lymphome, welches durch ein fast ausschließlich intravaskuläres Wachstum maligner Lymphozyten in kleinen Gefäßen, besonders Kapillaren, und eine schlechte Prognose charakterisiert ist. Das IVLBCL findet sich meist bei älteren Patienten mit reduziertem Allgemeinzustand, erhöhten Lactatdehydrogenase-Serumspiegeln, Anämie und Begleitsymptomen. In den meisten Fällen handelt es sich bei Diagnosestellung bereits um eine disseminierte Erkrankung. Interessanterweise scheint die klinische Manifestation von der geografischen Herkunft der Patienten abzuhängen. Fälle in den westlichen Ländern zeigen typischerweise eine Haut- und ZNS-Beteiligung, während Patienten aus asiatischen Ländern bevorzugt ein hämophagozytisches Syndrom und eine Knochenmarkbeteiligung aufweisen [12].

In $\odot$ Tab. 1 sind die epidemiologischen Daten und in $\triangle$ Tab. 2 die charakteristischen diagnostischen Merkmale der 3 Haupttypen kutaner B-Zell-Lymphome zusammenfassend aufgeführt.

$\mathrm{Zu}$ beachten ist, dass beim CBCL eine bcl-2-Expression und eine Translokation $t(14 ; 18)$ auf eine sekundäre Hautbeteiligung im Rahmen von nodalen Follikelzentrumslymphomen hinweisen kann, weiterhin korreliert eine bcl-2-Expression bei PCLBCL, LT mit einer schlechten Prognose $[1,4]$. Bei einer Manifestation des kutanen Keimzentrumslymphoms am Unterschenkel ist auch hier von einem insgesamt prognostisch ungünstigen Verlauf auszugehen. Neuere Untersuchungen konnten zeigen, dass die immunhistochemische Färbung für IgM an Paraffinschnitten als einfaches und nützliches zusätzliches Kriterium für die Unterscheidung zwischen PCLBCL, LT und PCFCL herangezogen werden kann [13].

Primär kutane B-Zell-Lymphome sind typischerweise Erkrankungen des höheren Erwachsenenalters, können jedoch auch zu Beginn des Erwachsenenalters auftreten.

\section{Ausbreitungsdiagnostik und TNM-basierte Stadieneinteilung $\nabla$}

Die Diagnostik beginnt mit einer Anamnese über Dauer, Art, Ausdehnung und Evolution der Hautmanifestationen. Die klinische Ganzkörperuntersuchung beinhaltet die Inspektion der Haut, die Palpation der peripheren Lymphknoten, Leber und Milz. Die Laboruntersuchungen beinhalten das komplette Routinelabor inklusive Differenzialblutbild und LDH, zusätzlich die (Immun-) Elektrophorese aus Serum und Urin. Bei Erstdiagnose eines $\mathrm{CBCL}$ empfehlen sich weiterhin eine Borrelien- und HIV-Serologie sowie die Bestimmung von Beta-2-Mikroglobulin. Eine durchfluss- 
zytometrische Analyse des peripheren Blutes sollte bei Auffälligkeiten im Blutbild erfolgen. Eine bildgebende Darstellung von zumindest Thorax, Abdomen und Becken sowie die Sonografie peripherer Lymphknoten sollte veranlasst werden. Typischerweise wird die kontrastmittelverstärkte Computertomografie (CT) als bildgebendes Verfahren gewählt. Es gibt zunehmend Evidenz für den Einsatz der Positronenemissionstomografie (PET) bzw. des Hybridverfahrens PET-CT für die Ausbreitungsdiagnostik und die Beurteilung des Therapieansprechens bei Lymphomen, sodass ihr Einsatz bei bestimmten Fragestellungen mit bedacht werden sollte $[14,15]$. Falls eine sichere CT nicht möglich ist, stehen alternativ das konventionelle Röntgen, die Sonografie oder die Magnetresonanztomografie (MRT) im Einzelfall zur Verfügung. Gemäß den standardisierten Kriterien für NHL sollten Lymphknoten mit einem Durchmesser von $>1 \mathrm{~cm}$ in der kurzen Achse oder signifikant erhöhter PET-Aktivität histologisch untersucht werden [14]. Eine Knochenmarkbiopsie und -aspirationszytologie ist bei PCLBCL, LT gefordert und wird bei den indolenten Formen, bei sonst unauffälligen Befunden, meist als optional angesehen. Es ist hier jedoch zu beachten, dass in einer Studie bei $11 \%$ der Patienten mit kutanem Follikelzentrumslymphom eine Knochenmarkbeteiligung festgestellt werden konnte und dies mit einer deutlich schlechteren Prognose einherging. Beim Marginalzonenlymphom mit primärer Manifestation an der Haut scheint eine Knochenmarkuntersuchung nur eine sehr eingeschränkte klinische Relevanz zu haben [4]. Zur Abgrenzung eines MALT-Lymphoms kann zusätzlich eine Ösophagogastroduodenoskopie erwogen werden.

Bei Vorhandensein von Begleitsymptomatik (Fieber $>38^{\circ} \mathrm{C}$, massiver Nachtschweiß, ungewollter Gewichtsverlust von mehr als $10 \%$ in 6 Monaten), anormalem Blutbild, erhöhtem LDH- oder Beta-2Mikroglobulin-Wert sollte der Verdacht auf ein systemisches NHL geäußert werden.

Bei Vorhandensein von Begleitsymptomatik, anormalem Blutbild, erhöhtem LDH- oder Beta-2-Mikroglobulin-Wert sollte der Verdacht auf ein systemisches NHL geäußert werden.

Die ISCL und die EORTC haben einen internationalen Konsensusvorschlag zur TNM-basierten Stadieneinteilung für alle primär kutanen Lymphome (mit Ausnahme der Mycosis fungoides und dem Sézary-Syndrom, für welche eine eigene Stadieneinteilung existiert) erarbeitet [14] ( Tab.3). Durch die Vereinheitlichung der Stadieneinteilung soll die Kommunikation über das Erkrankungsstadium, die Auswahl der passenden Therapieoptionen, die Standardisierung von Studieneinschlussund Therapieansprechkriterien und die Erhebung prospektiver Daten zur Überlebenswahrscheinlichkeit verbessert werden. Es ist wichtig festzuhalten, dass es sich bei der vorgeschlagenen

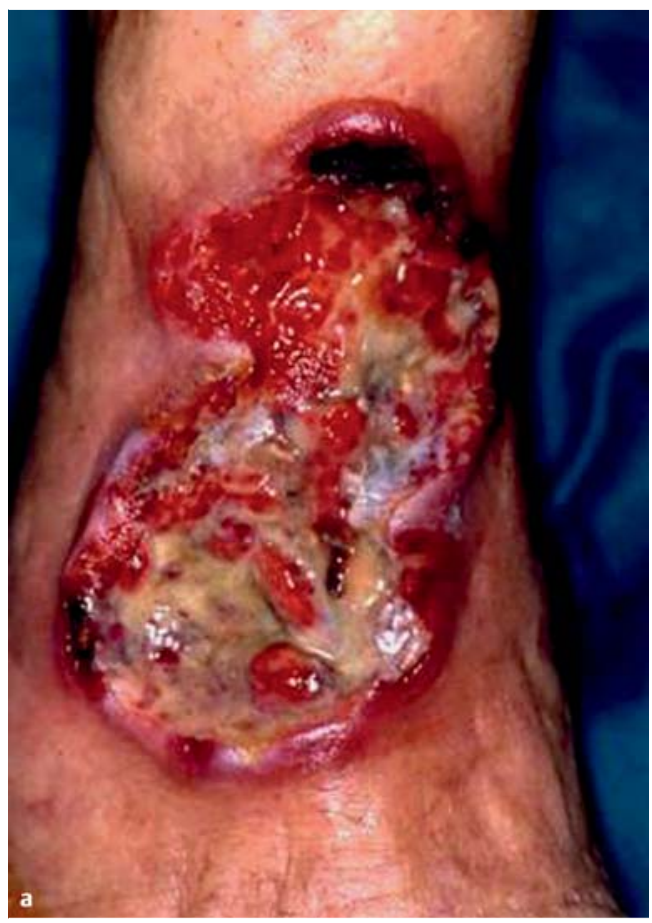

Abb. 3 Primär kutanes diffus-großzelliges B-Zell-Lymphom: Exulzerierter tumoröser Plaque am Unterschenkel (a), histologisch zeigen sich diffuse großzellige Infiltrate (b).

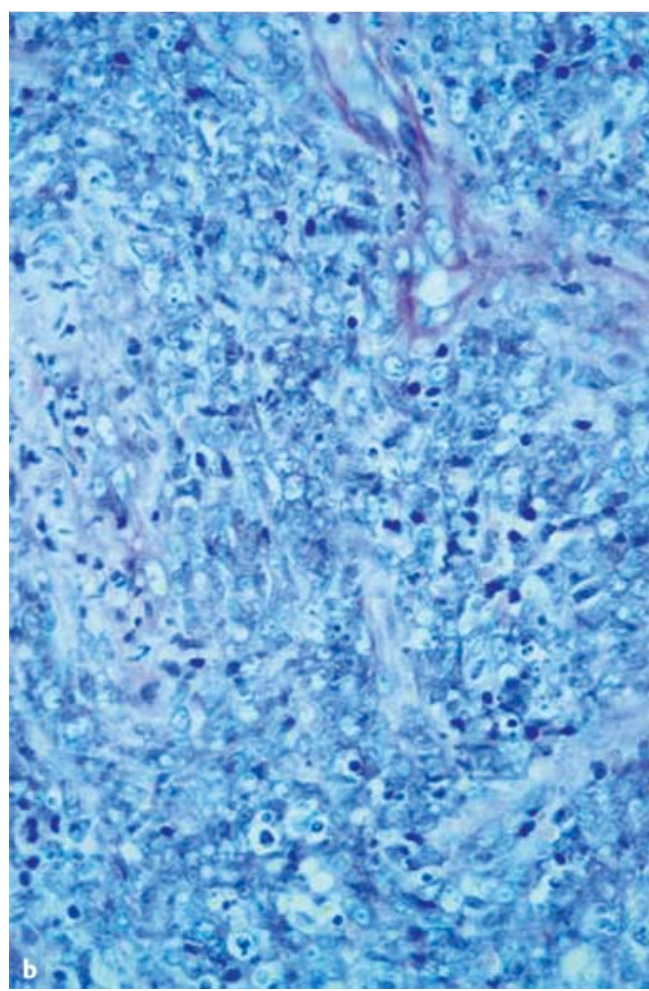

Tab. 1 Epidemiologische Daten primär kutaner B-Zell-Lymphome nach R. Fink-Puches et al. [6].

\begin{tabular}{|lllll} 
& $\begin{array}{l}\text { Anzahl } \\
\text { der Pa- } \\
\text { tienten }\end{array}$ & $\begin{array}{l}\text { Geschlechter- } \\
\text { verhältnis } \\
\text { (Männer:Frauen) }\end{array}$ & $\begin{array}{l}\text { mittleres } \\
\text { Alter in } \\
\text { Jahren } \\
\text { (Bereich) }\end{array}$ & $\begin{array}{l}\text { geschätzte } \\
\text { 5-Jahres- } \\
\text { Überlebens- } \\
\text { rate }\end{array}$ \\
\hline PCMZL & 62 & $1,69: 1$ & $55,5(17-86)$ & $98 \%$ \\
\hline PCFCL & 60 & $0,76: 1$ & $58,5(20-89)$ & $94 \%$ \\
\hline PCLBCL, LT & 23 & $0,76: 1$ & $72(43-86)$ & $58 \%$ \\
\hline
\end{tabular}


Tab. 2 Diagnostische Kennzeichen der 3 Haupttypen primär kutaner B-Zell-Lymphome.

\begin{tabular}{|c|c|c|c|}
\hline & PCMZL & PCFCL & PCLBCL, LT \\
\hline Histologie & $\begin{array}{l}\text { noduläre bis diffuse } \\
\text { Infiltrate bestehend } \\
\text { aus kleinen Lymphozy- } \\
\text { ten, Marginalzonen-B- } \\
\text { Zellen (zentrozyten- } \\
\text { ähnliche Zellen), } \\
\text { lymphoplasmazytoi- } \\
\text { den Zellen und Plasma- } \\
\text { zellen; reaktive Keim- } \\
\text { zentren sind häufig }\end{array}$ & $\begin{array}{l}\text { follikuläre, follikuläre } \\
\text { und diffuse oder } \\
\text { diffuse Infiltrate aus } \\
\text { neoplastischen Follikel- } \\
\text { zentrums-Zellen, ge- } \\
\text { wöhnlich bestehend } \\
\text { aus Zentrozyten und } \\
\text { einer variablen Anzahl } \\
\text { von Zentroblasten }\end{array}$ & $\begin{array}{l}\text { diffuses Infiltrat } \\
\text { mit einer Prädo- } \\
\text { minanz oder kon- } \\
\text { fluierende Lagen } \\
\text { aus Zentroblas- } \\
\text { ten und Immuno- } \\
\text { blasten }\end{array}$ \\
\hline \multirow[t]{3}{*}{ Phänotyp } & $\mathrm{BCl}-2^{+}$ & $\mathrm{BCl}-2^{-}$ & $\mathrm{BCl}-2^{+}$ \\
\hline & $\mathrm{BCl}-6^{-}$ & $\mathrm{Bcl}-6^{+}$ & $\mathrm{BCl}-6^{+(-)}$ \\
\hline & CD10 & $\mathrm{CD} 10^{ \pm}$ & CD10- \\
\hline Klinik & $\begin{array}{l}\text { isoliert/gruppiert oder } \\
\text { multifokal rötliche bis } \\
\text { violette Papeln, } \\
\text { Plaques oder Knoten } \\
\text { (Durchmesser } 1-3 \mathrm{~cm} \text { ) }\end{array}$ & $\begin{array}{l}\text { solitäre oder grup- } \\
\text { pierte (selten multi- } \\
\text { fokal) dermal-sub- } \\
\text { kutane rötlich-livide } \\
\text { Nodi, selten Ulzeration } \\
\text { (Durchmesser }>3 \mathrm{~cm} \text { ) }\end{array}$ & $\begin{array}{l}\text { solitäre oder } \\
\text { multiple rötlich- } \\
\text { livide Knoten und } \\
\text { Tumoren }\end{array}$ \\
\hline Lokalisation & Stamm und Arme & $\begin{array}{l}\text { behaarter Kopf, Nacken } \\
\text { oder Stamm, selten an } \\
\text { den Beinen }\end{array}$ & $\begin{array}{l}\text { Bein, meist unter- } \\
\text { halb des Knies }\end{array}$ \\
\hline
\end{tabular}

Tab. 3 ISCL/EORTC-Empfehlung zur TNM-Klassifikation für primär kutane Lymphome (nicht für Mycosis fungoides oder Sézary-Syndrom). Zur genauen Definition der Körperregionen wird auf die Originalliteratur verwiesen. Die Definition der Lymphknotenstationen entspricht dem Ann-ArborSystem.

\section{Klassifikation}

T1: solitärer Hautbefall

T1a: eine solitäre Läsion $<5 \mathrm{~cm}$ im Durchmesser

T1b: eine solitäre Läsion $>5 \mathrm{~cm}$ im Durchmesser

T2: regionaler Hautbefall: multiple Läsionen limitiert auf 1 oder 2 benachbarte Körperregionen

T2a: Gesamtheit der Herde befindet sich innerhalb einer Kreisfläche mit $<15 \mathrm{~cm}$ Durchmesser

T2b: Gesamtheit der Herde befindet sich innerhalb einer Kreisfläche mit $>15-$ und $<30 \mathrm{~cm}$ Durchmesser

T2c: Gesamtheit der Herde befindet sich innerhalb einer Kreisfläche mit $>30 \mathrm{~cm}$ Durchmesser

T3: generalisierter Hautbefall

T3a: multiple Läsionen in 2 nicht benachbarten Körperregionen

T3b: multiple Läsionen in $\geq 3$ Körperregionen

N

N0: keine klinische oder pathologische LK-Beteiligung

N1: Beteiligung einer peripheren LK-Region, welche ein Hautareal gegenwärtigen oder ehemaligen Befalls drainiert

N2: Beteiligung von 3 oder mehr peripheren LK-Regionen oder Beteiligung irgendeiner LK-Region, welche nicht ein gegenwärtig oder ehemals befallenes Hautareal drainiert N3: Beteiligung zentraler LK

M0: keine Evidenz für extrakutanen, extranodalen Befall

M1: extrakutaner, extranodaler Befall vorhanden

LK: Lymphknoten



Stadieneinteilung um eine rein anatomische Information über die Ausdehnung der Erkrankung handelt. Erste Evaluierungen konnten für das PCLBCL, LT eine gewisse prognostische Relevanz des neuen TNM-Systems zeigen, bei den indolenten Formen (PCFCL, PCMZL) zeigte sich keine Korrelation zwischen T-Stadium und krankheitsspezifischer 5-Jahres-Überlebenswahrscheinlichkeit [16].

\section{Therapie und Prognose}

Die Therapieempfehlungen der deutschen Leitlinie kutane Lymphome [17] und der EORTCISCL-Konsensusempfehlungen [4] basieren in erster Linie auf Fallberichten und kleinen retrospektiven Kohortenstudien, da randomisierte kontrollierte Studien fehlen.

Bei einer Minderheit von europäischen, nicht aber bei asiatischen oder US-amerikanischen Fällen von PCMZL konnte eine Assoziation zu Borrelia burgdorferi gefunden werden [18-21]. Analog zur Behandlung des gastralen MALT-Lymphoms mit Antibiotika zur Helicobacter-pylori-Eradikation, legen einige Übersichtsarbeiten nahe, das mit B. burgdorferi assoziierte PCMZL zuerst mit einer antibiotischen Therapie zu behandeln. Die Effizienz einer Antibiotikatherapie ist nur sehr spärlich dokumentiert. Nach den vorhandenen Daten kann in bis zu 40\% der Fälle mit einem Ansprechen gerechnet werden. Daten von 8 Patienten legen nahe, dass eine systemische Cephalosporintherapie einer oralen Therapie mit Doxycyclin überlegen ist [4]. Manche Autoren empfehlen unabhängig vom Borrelien-Status bei PCMZL und PCFCL eine antibiotische Therapie (3-wöchig Doxycyclin $100 \mathrm{mg}$ 2-mal/Tag) [3]. Die Auswahl der Therapieoptionen richtet sich in erster Linie nach der Klassifikation (indolente NHL vs. PCLBCL, LT) und der Verteilung (isolierte/gruppierte vs. multiple Herde). Eine Übersicht der in der deutschen Leitlinie kutane Lymphome empfohlenen Therapieoptionen findet sich in 8 Tab. 4 und 5.

Neue Studien zeigen, dass das PCFCL mit Lokalisation am Bein eine ähnlich schlechte Prognose hat wie das PCLBCL, LT. Und es erscheint gerechtfertigt, diese Patienten gemäß den Empfehlungen für das PCLBCL, LT zu behandeln $[4,22,23]$.

Die Auswahl der Therapieoptionen richtet sich in erster Linie nach der Klassifikation (indolente NHL vs. PCLBCL, LT) und der Verteilung (isolierte/gruppierte vs. multiple Herde).

Zur Erfolgsrate verschiedener Strahlenqualitäten und Dosierungen liegen nur wenig vergleichbare Daten vor. In der deutschen Leitlinie „Kutane Lymphome“ [17] werden eine Röntgenweichstrahltherapie 6-10 × 2 Gy oder schnelle Elektronen $40 \mathrm{~Gy}$ empfohlen. Alternativ kommen bei den indolenten Formen neben der Exzision intraläsional appliziertes Interferon alpha, ein Glukokortikosteroid oder Rituximab, einem gegen das BZell-Antigen CD20 gerichteten humanisierten Antikörper, in Betracht [17]. In einer retrospektiven Studie an 9 Patienten mit indolentem primär kutanem B-Zell-Lymphom wurde Rituximab 7-mal intraläsional und 2-mal systemisch verabreicht. Die Autoren kommen zu dem Schluss, dass Rituximab eine gut verträgliche und effektive Therapieoption bei indolenten primär kutanen B-Zell-Lymphomen darstellt. Während bei 
isolierten/gruppierten Herden eine intraläsionale Gabe erfolgen kann, sollte bei multiplen Herden die systemische Gabe (i.v.) erfolgen. Die Nebenwirkungen scheinen dabei mit der höheren intravenösen Dosis nicht zuzunehmen. Angemerkt wird zudem, dass eine intraläsionale Gabe die Behandlungskosten drastisch senkt [24]. Nach einer initialen Exzision oder Radiotherapie kann bei annähernd allen Patienten mit PCMZL oder PCFCL eine komplette Remission erreicht werden. Es muss jedoch bei ca. 40-50\% mit einem Rezidiv gerechnet werden. Die Radiotherapie bei PCLBCL, LT ist insgesamt weniger erfolgreich mit Ansprechraten von $88 \%$ und Rezidivraten von $58 \%$, meist außerhalb, aber auch innerhalb des Bestrahlungsfelds [4].

Zur Erhaltungstherapie können aufgrund fehlender klinischer Studien in der Leitlinie keine Angaben gemacht werden. Im Fall eines Rezidivs kann bei den niedrig malignen CBCL-Formen erneut eine Radiatio (bevorzugt bei Rezidiven außerhalb des Bestrahlungsfelds), eine Exzision oder eine Lokaltherapie der 2. Wahl erfolgen, ohne dass sich die Gesamtprognose verschlechtert. Eine Therapieeskalation mit Einleitung einer systemischen Therapie ist nicht automatisch notwendig, solange die anderen Optionen anwendbar sind [4]. Um die Rezidivwahrscheinlichkeit bei niedrig malignen CBCL-Formen besser abschätzen zu können, wurde kürzlich ein neuer prognostischer Index, der sogenannte CLIPI (cutaneous lymphoma international prognostic index), vorgeschlagen. Der CLIPI soll durch die Erhebung einiger klinischer Merkmale (LDH, Anzahl der Läsionen, Morphologie der Läsionen [nodulär oder nicht]) eine bessere risikoadaptierte Therapieauswahl ermöglichen [25].

Da das klinische Verhalten von PCLBCL, LT ähnlich den systemischen diffus großzelligen B-Zell-Lymphomen (DLBCL) ist, sollten diese im Fall multipler Herde primär systemisch behandelt werden. Die deutsche Leitlinie kutane Lymphome sieht hier als Therapie der 1 . Wahl eine Polychemotherapie kombiniert mit Rituximab vor (R-CHOP: Rituximab, Cyclophosphamid, Hydroxydoxorubicin, Vincristin, Prednisolon). Als Therapie der 2. Wahl bei isolierten/gruppierten oder multiplen Herden kommt eine Monotherapie mit pegyliertem liposomalen Doxorubicin in Betracht [17]. In einer aktuellen Arbeit konnten Pulini et al. bei 4 Patienten mit PCLBCL, LT zeigen, dass niedrig dosiertes $\left(20 \mathrm{mg} / \mathrm{m}^{2}\right)$ pegyliertes liposomales Doxorubicin (im Vergleich zur Standarddosis von $50 \mathrm{mg} / \mathrm{m}^{2}$ bei soliden Tumoren alle 4 Wochen) eine signifikante klinische Wirksamkeit bei einem guten Sicherheitsprofil hatte [26]. Die Patienten erhielten alle 3-4 Wochen $20 \mathrm{mg} / \mathrm{m}^{2}$ pegyliertes liposomales Doxorubicin begleitend zu täglich $300 \mathrm{mg}$ oralem Pyridoxin von Therapiebeginn bis 1 Monat nach Beendigung. Innerhalb von 3 Monaten erreichten alle Patienten eine Vollremission mit einer dokumentierten Dauer bis max. 69 Monaten. Erfreuli-
Tab. 4 Therapieempfehlungen bei indolenten primär kutanen B-Zell-Lymphomen (Keimzentrumslymphom, Marginalzonenlymphom).

\begin{tabular}{llll} 
Ausdehnung & $\begin{array}{l}\text { Therapie der } \\
\text { 1. Wahl }\end{array}$ & $\begin{array}{l}\text { Therapie der } \\
\text { 2. Wahl }\end{array}$ \\
$\begin{array}{l}\text { isolierte/gruppierte } \\
\text { Herde }\end{array}$ & $\begin{array}{l}\text { vorgeschaltete } \\
\text { Antibiotika- } \\
\text { therapie } \\
\text { (falls borrelien- } \\
\text { assoziiert) }\end{array}$ & $\begin{array}{l}\text { Totalexzision } \\
\text { Radiotherapie }\end{array}$ & $\begin{array}{l}\text { intraläsional Rituximab } \\
\text { intraläsional IFN- } \alpha \\
\text { intraläsional Steroid }\end{array}$ \\
\hline multiple Herde & Radiotherapie & $\begin{array}{l}\text { intraläsional IFN- } \alpha \\
\text { intraläsional Rituximab } \\
\text { i.v. Rituximab }\end{array}$ \\
\hline
\end{tabular}

IFN: Interferon

Tab. 5 Therapieempfehlungen beim primär kutanen diffus großzelligen B-Zell-Lymphom (leg type).

\begin{tabular}{lll}
\hline Ausdehnung & Therapie der 1. Wahl & Therapie der 2. Wahl \\
\hline $\begin{array}{l}\text { isolierte/gruppierte } \\
\text { Herde }\end{array}$ & $\begin{array}{l}\text { Radiotherapie } \\
\text { Exzision }\end{array}$ & $\begin{array}{l}\text { Monochemotherapie, z. B. } \\
\text { liposomales Doxorubicin }\end{array}$ \\
\hline multiple Herde & Polychemotherapie + Rituximab & \\
\hline
\end{tabular}

cherweise kam es unter der niedrigen Dosis und der Pyridoxin-Begleittherapie nicht zum oft therapielimitierenden palmoplantaren Erythrodysästhesie-Syndrom. Interessanterweise führte die Dosissteigerung auf $40 \mathrm{mg} / \mathrm{m}^{2}$ zu erhöhter Toxizität, nicht jedoch zu einem besseren Ansprechen. Für die Behandlung des DLBCL beim älteren Patienten zeigte sich die Kombinationsbehandlung von Rituximab mit der auf dem Anthrazyklin Doxorubicin basierten Polychemotherapie CHOP effektiver als CHOP allein. Die bcl-2-Expression beim DLBCL ist mit einer schlechteren Prognose und Therapieversagen assoziiert. Die Kombinationstherapie mit Rituximab scheint hier die bcl2-assoziierte Chemotherapieresistenz zu durchbrechen [27]. Rituximab bewirkt auch eine Steigerung der radiatiogetriggerten Apoptose bei NHL und erhöht dadurch die Radiosensitivität $[28,29]$. Es kann somit im individuellen Fall auch vorteilhaft sein, eine Radiotherapie mit einer Rituximab-Vortherapie zu kombinieren. Im Fall eines Rezidivs wird beim PCLBCL, LT empfohlen, dass diese Patienten gemäß der lokal üblichen hämatologischen Protokolle für rezidivierte diffus-großzellige B-Zell-Lymphome behandelt werden [4].

Therapie der 1 . Wahl bei den 3 Hauptformen primär kutaner B-Zell-Lymphome ist in der Regel die Exzision und/oder die Radiatio.

\section{Nachsorgeempfehlungen}

$\nabla$

Evidenzbasierte Nachsorgeempfehlungen für CBCL liegen nicht vor [17]. Wir empfehlen zur Orientierung beim CBCL im 1.-3. Jahr ein 3-monatiges Nachsorgeintervall mit Ganzkörperinspektion der Haut und Palpation von Lymphknoten, Leber und Milz, Blut- und bildgebende Unter- 
suchungen nach individuellem Bedarf, im 4.-5. Jahr klinische Kontrollen alle 6 Monate, ab dem 6. Jahr klinische Kontrollen einmal jährlich.

\section{Zusammenfassung}

20 - 25\% der primär kutanen Lymphome können den primär kutanen B-Zell-Lymphomen zugeordnet werden. Die 2005 eingeführte WHO-EORTCKlassifikation kutaner Lymphome berücksichtigt 3 Haupttypen von primär kutanen B-Zell-Lymphomen:

- primär kutane Marginalzonen B-Zell-Lymphome (PCMZL)

- primär kutane Keimzentrumslymphome (PCFCL)

- primär kutane diffus-großzellige B-Zell-Lymphome (leg type) (PCLBCL, LT)

Das PCMZL und das PCFCL zählen mit einer 5-Jahres-Überlebensrate von $\geq 95 \%$ zu den indolenten Non-Hodgkin-Lymphomen. Das PCLBCL, LT unterscheidet sich hiervon grundsätzlich mit einer 5Jahres-Überlebensrate von nur etwa 55\% (intermediärer Typ). Für die Durchuntersuchung und TNM-basierte Stadieneinteilung für kutane BZell-Lymphome unterbreiteten ISCL und EORTC im Jahr 2006 einen Vorschlag, der jedoch in erster Linie die anatomisch Ausbreitung berücksichtigt und nur eine sehr begrenzte prognostische Aussagekraft besitzt. Von besonderer Wichtigkeit bei kutanen B-Zell-Lymphomen ist die Abgrenzung von primär kutanen Lymphomen zu sekundärem Hautbefall bei nodalen oder MALT-Lymphomen. Die Auswahl der Therapieoptionen richtet sich in erster Linie nach der Klassifikation (indolente NHL vs. PCLBCL, LT) und der Verteilung (isolierte/ gruppierte vs. multiple Herde). Als wichtigste Therapieoption bei isolierten/gruppierten Herden aller Formen von kutanen B-Zell-Lymphomen gilt die Totalexzision und/oder Radiatio. Bei den indolenten Formen kann bei multiplen Herden oder im Fall eines Rezidivs weiterhin mit einer hautgerichteten Therapieform gearbeitet werden, alternativ steht eine systemische Immuntherapie mit Rituximab zur Verfügung. Beim PCLBCL, LT wird bei multiplen Herden oder im Fall eines Rezidivs eine Polychemotherapie plus Rituximab empfohlen.

Interessenkonflikt: Die Autoren geben an, dass keine Interessenkonflikte bestehen.

\section{Literatur}

1 Willemze R, Jaffe ES, Burg G et al. WHO-EORTC classification for cutaneous lymphomas. Blood 2005; 105: 3768 3785

2 Assaf C, Gellrich S, Steinhoff $M$ et al. Cutaneous lymphomas in Germany: an analysis of the Central Cutaneous Lymphoma Registry of the German Society of Dermatology (DDG). J Dtsch Dermatol Ges 2007; 5: 662 - 668

3 Dummer R, Stadler R, Sterry W. Cutaneous lymphomas. J Dtsch Dermatol Ges 2007; 5: 605-617

4 Senff NJ, Noordijk EM, Kim YH et al. European Organization for Research and Treatment of Cancer; International Society for Cutaneous Lymphoma. European Organization for Research and Treatment of Cancer and International Society for Cutaneous Lymphoma consensus recommendations for the management of cutaneous B-cell lymphomas. Blood 2008; 112: 1600-1609

5 Burg G, Kempf W, Cozzio A et al. Cutaneous malignant lymphomas: update 2006. J Dtsch Dermatol Ges 2006; 4: $914-933$

6 Fink-Puches R, Zenahlik P, Bäck B et al. Primary cutaneous lymphomas: applicability of current classification schemes (European Organization for Research and Treatment of Cancer, World Health Organization) based on clinicopathologic features observed in a large group of patients. Blood 2002; 99: 800-805

7 Child FJ, Woollons A, Price ML et al. Multiple cutaneous immunocytoma with secondary anetoderma: a report of two cases. Br J Dermatol 2000; 143: 165 - 170

8 de Leval L, Harris NL, Longtine J et al. Cutaneous b-cell lymphomas of follicular and marginal zone types: use of $\mathrm{BCl}$ 6, CD10, BCl-2, and CD21 in differential diagnosis and classification. Am J Surg Pathol 2001; 25: 732 - 741

9 Swerdlow SH, Campo E, Harris NL et al. World Health Organization classification of tumours of haematopoietic and lymphoid tissues. Lyon: IARC Press; 2008

10 Grange F, Petrella T, Beylot-Barry M et al. Bcl-2 protein expression is the strongest independent prognostic factor of survival in primary cutaneous large B-cell lymphomas. Blood 2004; 103: 3662-3668

11 Hallermann C, Niermann C, Fischer RJ et al. New prognostic relevant factors in primary cutaneous diffuse large Bcell lymphomas. J Am Acad Dermatol 2007; 56: 588 597

12 Ponzoni M, Ferreri AJ, Campo E et al. Definition, diagnosis, and management of intravascular large B-cell lymphoma: proposals and perspectives from an international consensus meeting. J Clin Oncol 2007; 25: 3168-3173

13 Koens L, Vermeer MH, Willemze R et al. IgM expression on paraffin sections distinguishes primary cutaneous large B-cell lymphoma, leg type from primary cutaneous follicle center lymphoma. Am J Surg Pathol 2010; 34: 1043 $-1048$

14 Kim YH, Willemze R, Pimpinelli N et al. TNM classification system for primary cutaneous lymphomas other than mycosis fungoides and Sezary syndrome: a proposal of the International Society for Cutaneous Lymphomas (ISCL) and the Cutaneous Lymphoma Task Force of the European Organization of Research and Treatment of Cancer (EORTC). Blood 2007; 110: 479-484

15 Cronin CG, Swords R, Truong MT et al. Clinical utility of PET/CT in lymphoma. Am J Roentgenol 2010; 194: W91 $-\mathrm{W} 103$

16 Senff $N$, Willemze $R$. The applicability and prognostic value of the new TNM classification system for primary cutaneous lymphomas other than mycosis fungoides and Sézary syndrome: results on a large cohort of primary cutaneous B-cell lymphomas and comparison with the system used by the Dutch Cutaneous Lymphoma Group. Br J Dermatol 2007; 157: 1205-1211

17 Stadler R, Assaf C, Klemke CD et al. Short German guidelines: cutaneous lymphomas. J Dtsch Dermatol Ges 2008; 6 (Suppl. 1): S25-S31 
18 Cerroni L, Zöchling N, Pütz B et al. Infection by Borrelia burgdorferi and cutaneous B-cell lymphoma. J Cutan Pathol 1997; 24: 457-461

19 Goodlad IR, Davidson MM, Hollowood K et al. Primary cutaneous B-cell lymphoma and Borrelia burgdorferi infection in patients from the Highlands of Scotland. Am J Surg Pathol 2000; 24: 1279-1285

$20 \mathrm{Li} \mathrm{C}$, Inagaki H, Kuo TT et al. Primary cutaneous marginal zone B-cell lymphoma: a molecular and clinicopathologic study of 24 asian cases. Am J Surg Pathol 2003; 27: 1061 - 1069

21 Wood GS, Kamath NV, Guitart I et al. Absence of Borrelia burgdorferi DNA in cutaneous B-cell lymphomas from the United States. J Cutan Pathol 2001; 28: $502-507$

22 Senff NJ, Hoefnagel Jj, Jansen PM et al. Reclassification of 300 primary cutaneous B-Cell lymphomas according to the new WHO-EORTC classification for cutaneous lymphomas: comparison with previous classifications and identification of prognostic markers. J Clin Oncol 2007; 25: 1581-1587

23 Kodama K, Massone C, Chott A et al. Primary cutaneous large B-cell lymphomas: clinicopathologic features, classification, and prognostic factors in a large series of patients. Blood 2005; 106: 2491-2497
24 Fink-Puches $R$, Wolf $I H$, Zalaudek I et al. Treatment of primary cutaneous Bcell lymphoma with rituximab. J Am Acad Dermatol 2005; 52: 847-853

25 Mian M, Marcheselli L, Luminari S et al. CLIPI: a new prognostic index for indolent cutaneous B cell lymphoma proposed by the International Extranodal Lymphoma Study Group (IELSG 11). Ann Hematol [in press]

26 Pulini S, Rupoli S, Goteri G et al. Efficacy and safety of pegylated liposomal doxorubicin in primary cutaneous B-cell lymphomas and comparison with the commonly used therapies. Eur J Haematol 2009; 82: 184-193

27 Mounier N, Briere J, Gisselbrecht C et al. Rituximab plus CHOP (R-CHOP) overcomes bcl-2-associated resistance to chemotherapy in elderly patients with diffuse large B-cell lymphoma (DLBCL). Blood 2003; 101: 4279-4284

28 Skvortsova I, Skvortsov S, Popper BA et al. Rituximab enhances radiation-triggered apoptosis in non-Hodgkin's lymphoma cells via caspase-dependent and - independent mechanisms. J Radiat Res (Tokyo) 2006; 47: 183- 196

29 Skvortsova I, Popper BA, Skvortsov $S$ et al. Pretreatment with rituximab enhances radiosensitivity of non-Hodgkin's lymphoma cells. J Radiat Res (Tokyo) 2005; 46: 241 - 248

\section{CME-Fortbildung mit der Aktuellen Dermatologie}

Zertifizierte Fortbildung Hinter der Abkürzung CME verbirgt sich „continuing medical education“, also kontinuierliche medizinische Fort- und Weiterbildung. Zur Dokumentation der kontinuierlichen Fortbildung der Ärzte wurde das Fortbildungszertifikat der Ärztekammern etabliert. Hauptzielgruppe für das Fortbildungszertifikat sind Ärzte mit abgeschlossener Facharztausbildung, die im 5-jährigen Turnus einen Fortbildungsnachweis erbringen müssen. Es ist jedoch auch für Ärzte im Praktikum bzw. in der Facharztweiterbildung gedacht.

Die Fortbildungseinheit In den einheitlichen Bewertungskriterien der Bundesärztekammer ist festgelegt: „Die Grundeinheit der Fortbildungsaktivitäten ist der Fortbildungspunkt. Dieser entspricht in der Regel einer abgeschlossenen Fortbildungsstunde (45 Minuten)“. Für die erworbenen Fortbildungspunkte muss ein Nachweis erbracht werden. Hat man die erforderliche Anzahl von 250 Punkten gesammelt, kann man das Fortbildungszertifikat bei seiner Ärztekammer beantragen, welches man wiederum bei der KV (niedergelassene Ärzte) oder bei seinem Klinikträger (Klinikärzte) vorlegen muss.

Anerkennung der CME-Beiträge Die Fortbildung in der Aktuellen Dermatologie wurde von der Nordrheinischen Akademie für ärztliche Fort- und Weiterbildung für das Fortbildungszertifikat anerkannt, das heißt, die Vergabe der Punkte kann direkt durch die Thieme Verlagsgruppe erfolgen. Die Fortbildung in der Aktuellen Dermatologie gehört zur Kategorie „strukturierte interaktive Fortbildung“. Entsprechend einer Absprache der Ärztekammern werden die von der Nordrheinischen Akademie für ärztliche Fort- und Weiterbildung anerkannten Fortbildungsveranstaltungen auch von den anderen zertifizierenden Ärztekammern anerkannt.
Datenschutz Ihre Daten werden ausschließlich für die Bearbeitung dieser Fortbildungseinheit verwendet. Es erfolgt keine Speicherung der Ergebnisse über die für die Bearbeitung der Fortbildungseinheit notwendige Zeit hinaus. Die Daten werden nach Versand der Testate anonymisiert. Namens- und Adressangaben dienen nur dem Versand der Testate. Die Angaben zur Person dienen nur statistischen Zwecken und werden von den Adressangaben getrennt und anonymisiert verarbeitet.

Teilnahme Jede Ärztin und jeder Arzt soll das Fortbildungszertifikat erlangen können. Deshalb ist die Teilnahme am CME-Programm der Aktuellen Dermatologie nicht an ein Abonnement geknüpft! Die Teilnahme ist im Internet unter http://cme.thieme. de möglich. Man muss sich registrieren, wobei die Teilnahme an Fortbildungen abonnierter Zeitschriften ohne Zusatzkosten möglich ist.

Teilnahmebedingungen Für eine Fortbildungseinheit erhalten Sie 3 Fortbildungspunkte im Rahmen des Fortbildungszertifikates. Hierfür müssen $70 \%$ der Fragen richtig beantwortet sein.

CME-Fortbildung für Nicht-Abonnenten Teilnehmer, die nicht Abonnenten der Aktuellen Dermatologie sind, können für die Internet-Teilnahme dort direkt ein Guthaben einrichten, von dem pro Teilnahme ein Unkostenbeitrag abgebucht wird.

Teilnahme online unter http://cme.thieme.de 


\section{CME-Fragen Primär kutane B-Zell-Lymphome}

1 Die geschätzte Inzidenz (Neuerkrankungen pro Einwohner und Jahr) der primär kutanen Non-Hodgkin-Lymphome beträgt:

$\begin{array}{ll}\text { A } & 1 / 1000 \\ \text { B } & 1 / 100000 \\ \text { C } & 1 / 10000 \\ \text { D } & 1 / 100 \\ \text { E } & 1 / 1000000\end{array}$

Das primär kutane Marginalzonen B-Zell-Lymphom

A hat eine indolente klinische Verlaufsform mit einer 5-Jahres-Überlebensrate von 75\%.

B zeigt häufig ein aggressives klinisches Verhalten.

C hat eine indolente klinische Verlaufsform mit einer 5-Jahres-Überlebensrate von $\geq 95 \%$.

D hat eine intermediäre klinische Verlaufsform mit einer 5-Jahres-Überlebensrate von 55\%.

E hat eine ähnliche Prognose wie das diffus-großzellige B-Zell-Lymphom der unteren Extremität.

A bilden etwa $40 \%$ der kutanen Lymphome.

B werden von WHO und EORTC ohne Berücksichtigung ihres klinischen Verhaltens klassifiziert.

C sind immer zweifelsfrei von Pseudolymphomen zu unterscheiden.

D bilden etwa 5\% der kutanen Lymphome.

E können auch ein intravaskuläres Wachstumsmuster zeigen.

\section{4}

A treten ausschließlich im hohen Erwachsenenalter auf.

B ulzerieren häufig.

C treten bevorzugt am Stamm oder den Armen auf.

D zeigen häufig eine extrakutane Beteiligung.

E zeigen nur äußerst selten ein kutanes Rezidiv.

A zeigen immer ein follikuläres Wachstumsmuster.

B können ein diffuses Wachstumsmuster zeigen.

C exprimieren Pan-T-Zell-Antigene.

D treten meist um das 40. Lebensjahr auf.

E sind meistens bcl-6-negativ.
6

Primär kutane B-Zell-Lymphome können nach einem internationalen Konsensusvorschlag (ISCL/EORTC) in Stadien nach dem TNM-System eingeteilt werden. Welche Aussage ist richtig?

A Beim Marginalzonen-Lymphom korreliert das T-Stadium eng mit der Prognose.

B Die genannte Stadieneinteilung kann nur bei B-ZellLymphomen angewendet werden.

C Es handelt sich in erster Linie um eine anatomische Information über die Erkrankungsausdehnung.

D T3 ist definiert als solitärer Hautbefall.

E N1 ist definiert als Beteiligung zentraler Lymphknoten.

7

A kann nur intraläsional verabreicht werden.

B ist ein Antikörper gegen das B-Zell-Antigen CD20.

C ist ein TNF-alpha-Antagonist.

D kommt nur als Monotherapie zur Anwendung.

E verschlechtert die radiatiogetriggerte Apoptose bei Lymphomen.

A eine Polychemotherapie.

B ein seltenes B-Zell-Lymphom.

C einen prognostischen Index für niedrig maligne kutane B-Zell-Lymphome.

D einen multimodalen Therapieansatz.

E ein B-Zell-Antigen.

Für die Therapie des kutanen Marginalzonen-Lymphoms gilt:

Liposomales Doxorubicin gilt als Therapie der 1. Wahl.

B Das initiale Ansprechen ist meist sehr schlecht.

C Die Exzision und/oder Radiatio gelten als Therapie der 1. Wahl.

D Kutane Rezidive kommen praktisch nicht vor.

E Ein Rezidiv verschlechtert immer die Gesamtprognose.

\section{0}

Die Begleitsymptomatik

A beinhaltet v.a. Übelkeit und Erbrechen.

B ist bei kutanen Lymphomen nicht von Bedeutung.

C ist typisch für das Follikelzentrumslymphom.

D kann ein Hinweis auf ein systemisches Lymphom sein.

E beinhaltet nicht den Gewichtsverlust. 